\title{
EXCESO DE HORAS DE TRABAJO Y LA SALUD DEL DOCENTE EN EDUCACIÓN SUPERIOR
}

\section{EXCESSIVE WORKING HOURS AND HEALTH OF TEACHERS IN HIGHER EDUCATION}

\author{
Walter F. Deza Loyaga* \\ Jorge A. Aparicio Ballena**
}

\begin{abstract}
RESUMEN
L a presente investigación trata sobre los riesgos o daño a la salud del docente que trabaja dictando horas de clases en exceso y en más de un centro de trabajo, se escogió una muestra de docentes que trabajan a tiempo completo en un instituto superior y a la vez a tiempo parcial en diversas universidades. Estableciendo como objetivo, el determinar la relación que existe entre el exceso de horas de trabajo en la docencia y el daño a la salud del docente. La investigación se realizó entre los meses de abril y julio del 2016, a docentes que laboran en un instituto superior y universidades en forma paralela, a todos los docentes se aplicó una encuesta presencial con la finalidad de poder atender alguna observación pertinente. Mediante la observación directa se recogió información sobre las condiciones donde laboran los docentes. La muestra de trabajo corresponde 134 docentes de institutos y universidades. De la investigación se deduce que las horas en exceso de trabajo tiene relación directa con la mala alimentación durante la jornada, así como laborar en más de un centro de trabajo por las bajas remuneraciones que perciben, con la exigencia ergonómica traducida en levantar la voz permanentemente durante las clases, asociada al grado de exigencia del trabajo docente. Por lo que se concluye, que los docentes se encuentran en un nivel alto de exposición a factores de riesgos o daño de su salud, teniendo enfermedades diagnosticadas.
\end{abstract}

Palabras clave: enfermedades diagnosticadas, exceso de trabajo, exigencia ergonómica, factores de riesgo

* Ingeniero industrial egresado de la Universidad José Faustino Sánchez Carrión. Docente de la Universidad Católica de Santa María y de Senati. Correo electrñonico: wdeza@hotmail.com

** Licenciado en Administración de la Universidad de San Martín de Porres. Docente de la Universidad San Pedro, IFB Certus, Inverconsulting SA. Correo electrónico: jaba_91@hotmail.com 
ABSTRACT

This research is done by making an approach to the risks or harm health of teachers working dictating school hours in excess and in more than one workplace, a sample of teachers working full time at a higher institute was chosen as while part-time at various universities. Setting a target, to determine the relationship between excessive hours of work in teaching and damage to the health of teachers. The research was conducted between April and July 2016, teachers who work at a higher institute and universities in parallel, all teachers a face survey with the aim of addressing any relevant observation was applied. Through direct observation about the conditions where they work teachers collected. The sample of 134 teachers work corresponds colleges and universities. Research shows that the hours overwork is directly related to poor diet during the day and work in more than one workplace by low pay they receive, with ergonomic requirements translated to raise his voice permanently during classes, associated with the level of demand of teaching. So it is concluded that teachers are at a high level of exposure to risk factors or damage your health, having diagnosed diseases.

Keywords: Diagnosed Diseases, Ergonomic Requirements, Overwork, Risk Factors

\section{INTRODUCCIÓN}

En un estudio de la Unesco (2015) sobre condiciones de trabajo y salud docente se refiere que:

[...] existe una relación directa en las condiciones de trabajo y salud respecto al rendimiento laboral. Así, los investigadores recalcan que la visión de la docencia como apostolado implica un sacrificio y renuncia que termina impactando al entorno profesional y familiar de los docentes. El mismo informe señala que un 42 por ciento de los profesores ha sufrido algún tipo de enfermedad psicológica durante su ejercicio.

Es conocido el dicho "todo en exceso es dañino". En este caso, el exceso de trabajo por parte del docente no escapa a esta máxima. Muchas veces buscamos obtener mayores ingresos poniendo en riesgo nuestra salud. La importancia del presente trabajo radica en que a partir de los datos tomados y obtenidos en la investigación, busquemos crear conciencia en la comunidad docente como en la alta dirección de instituciones educativas, que si queremos mejorar la calidad educativa debemos empezar por conocer al docente, tener una plana docente en buen estado de salud garantizará la calidad de la enseñanza que tanto se aspira conseguir, recordar que los docentes son responsables de formar personas quienes más tarde conducirán los destinos de muchas organizaciones donde les toque desempeñarse.

Nos hacemos la siguiente pregunta ¿Por qué los docentes trabajan en más de un centro de estudios, sabiendo que todo trabajo en exceso puede ocasionarles trastornos a la salud? Tal vez la respuesta del docente sería no me importa. Entonces si difundimos los resultados de la investigación donde corroboramos que trabajar en exceso genera problemas a la salud del docente, podríamos iniciar un cambio y regular la cantidad de horas de dictado, en el caso de la investigación prevalece la norma 80/20, que significa $80 \%$ de horas dictadas y $20 \%$ de preparación de clases en jornadas a tiempo completo, 
pero si a esto se suma el trabajo a tiempo parcial en otras instituciones educativas en forma paralela algunos docentes podrían llegar a más 60 horas por semana.

Previamente a la investigación, se han revisado similares estudios en el Perú, aunque existen pocos en torno a la relación entre el exceso de horas de trabajo y la salud docente, se destacan los trabajos de Soria y Chiroque (2004), Cuenca y Portocarrero (2003) y Fernández (2002), quienes corroboran que los docentes trabajan en forma desmedida con el afán de incrementar sus ingresos económicos y en otros casos buscando su autorrealización personal. A esto se asocia que la educación en la actualidad es un medio de ocupación para muchos profesionales.

La investigación se justifica porque obedece a un principio de la sociedad democrática que es garantizar la calidad de vida para toda su población, en este caso la responsabilidad recae en el estado y, por otro lado, la responsabilidad del profesorado tiene que ver directamente con el aprendizaje de los estudiantes, con la calidad de la educación y con las oportunidades de desarrollo del país. Pero también hay factores poco explorados que inciden en el desempeño profesional como por ejemplo las condiciones de trabajo (no es parte de la investigación), las cuales, unidas a otras determinantes, definen perfiles de salud-enfermedad para los docentes.

Esta investigación es el inicio y la intención es seguir profundizando los estudios con la finalidad de mejorar las condiciones laborales y asimismo determinar enfermedades profesionales para el docente, que en nuestro país aún no están legisladas. Dejamos el siguiente concepto del Sindicato Unificado de Trabajadores de la Educación de Buenos Aires (SUTEBA) (2) para reflexión:

Enfermedad profesional implica un daño en la salud del trabajador expuesto a ciertos riesgos laborales. Hay una relación directa entre riesgo laboral y daño producido, lo que implica que las condiciones de trabajo no son adecuadas y dañan.

En tal sentido, en la presente investigación buscamos encontrar si existe relación entre el exceso de horas de trabajo en la docencia y el daño a la salud del docente. Para dar respuesta a la investigación se ha establecido el objetivo siguiente: Determinar la relación que existe entre el exceso de horas de trabajo en la docencia y el daño a la salud del docente. Para conseguir el objetivo general, nos hemos planteado los siguientes objetivos específicos: 1. Identificar cuántas horas a la semanas trabajan. 2. Conocer las principales enfermedades diagnosticadas de los docentes. 3. Identificar por qué trabajan en más de un centro de trabajo.

\section{MÉTODOS}

El tipo de investigación es descriptiva, la población son docentes de un instituto técnico superior y universidades, la población total es de 210 docentes.

Para el cálculo de la muestra, se utilizó la fórmula:

$$
n=\frac{k^{\wedge} 2 * p^{*} q^{*} N}{\left(e^{\wedge 2 *}(N-1)\right)+k^{\wedge} 2 * p^{*} q}
$$


Donde:

$\mathrm{N}$ = población: 210 docentes

$\mathrm{K}=$ nivel de confianza: $1.96=95 \%$

$\mathrm{e}=$ margen de error: $5 \%$

$\mathrm{p}=$ proporción de docentes que poseen la característica del estudio: 0.4

$\mathrm{q}$ = proporción de docentes que no poseen la característica del estudio: 0.6

$\mathrm{n}=134$ encuestas

La población fue de 210 docentes de un instituto técnico superior y universidades, de los cuales el $40 \%$ son tiempo completo (TC) y el $60 \%$ a tiempo parcial contratados (TPC), la muestra estuvo formada 134 docentes, 88 docentes a TPC; 25 docentes a TC de institutos; y 21 docentes a TPC de universidades, la muestra estuvo conformada por 36 mujeres y 98 varones.

La investigación se basa en un trabajo de campo, con el que se pretende establecer grado de correlación entre el exceso de horas de trabajo en la docencia y el daño a la salud del docente, las variables se muestran en la Tabla 1.

Tabla 1. Caracterización de las variables

\begin{tabular}{l}
\hline \multicolumn{1}{c}{ Variables sociodemográficas } \\
\hline Sexo \\
Edad del docente \\
Descanso entre hora y hora de trabajo \\
Razones de trabajar en más un centro \\
Grado de exigencia del trabajo docente \\
Alimentación durante el trabajo \\
$\quad$ Variables de estudio \\
Horas trabajadas durante la semana \\
Trabaja en más centro de trabajo \\
Exigencia ergonómica durante las clases \\
Resultados de exámenes ocupacionales \\
Principales enfermedades diagnosticadas
\end{tabular}

\section{RESULTADOS}

De los datos recogidos de la muestra como variables sociodemográficas tenemos los siguientes resultados:

1) Distribución de trabajadores encuestados según sexo. La muestra del estudio corresponde a un $77 \%$ de sexo masculino y a un $33 \%$ de sexo femenino.

2) Distribución de los docentes según edad. Casi las 3/4 partes de los docentes tienen más de 41 años. La muestra arroja los siguientes datos: el rango de 20-30 años representa el 4\%, el rango de 31-40 años representa el $22 \%$, el rango de 41 a 50 años representa el $40 \%$, el rango de 51-60 años representa el $27 \%$, y el rango de 61 a más representa el $7 \%$. 
3) Tiempo de descanso entre hora y hora durante dictado de clases. La muestra arroja resultados sorprendentes, pues el $31 \%$ de los docentes no tienen descanso alguno y trabajan de corrido; el $22 \%$ de los docentes al menos descansa 5 minutos; el $34 \%$ de los docentes descansa entre 6 y 15 minutos, y solo el $13 \%$ de los docentes descansa más de 15 minutos.

4) Razones por las que trabajan en más de un centro de trabajo. La muestra arroja un contundente resultado, pues el $90 \%$ de los docentes trabaja en más de un centro de trabajo porque las remuneraciones son bajas. Un $4 \%$ de los docentes trabaja en más de un centro de trabajo porque le gusta enseñar y un $6 \%$ de los docentes trabaja en más de un centro de trabajo porque dispone de tiempo.

5) Grado de exigencia del trabajo docente. De los resultados de la muestra, el $54 \%$ considera que el grado de exigencia del trabajo docente es media; un $28 \%$ considera que el grado de exigencia del trabajo docente es alta y solo un $18 \%$ considera que el grado de exigencia del trabajo docente es baja.

6) Alimentación durante el trabajo. La muestra arroja que el $69 \%$ de los docentes toman sus alimentos fuera de casa, un $11 \%$ de los docentes lleva su refrigerio al trabajo, también un $11 \%$ de los docentes toma sus alimentos unos días en casa y otros en la calle, solo el $9 \%$ de los docentes manifestó que toma sus alimentos en casa.

En esta parte se presentan los resultados de la investigación que corresponden a las variables del estudio:

1) Horas trabajadas durante la semana. La gran mayoría de los docentes, que es el $82 \%$, manifiesta que trabaja más de 40 horas durante la semana, el restante 18\% manifiesta que trabaja entre 31 a 40 horas durante la semana. Como lo podemos apreciar en la tabla 2.

Tabla 2. Horas trabajadas durante la semana

\begin{tabular}{lcc}
\hline Horas trabajadas & Frecuencia & $\%$ \\
\hline Menos de 20 horas & 0 & 0 \\
Entre 20 a 30 horas & 0 & 0 \\
Entre 31 a 40 horas & 24 & 18 \\
Más de 40 horas & 110 & 82 \\
Total & 134 & 100 \\
\hline
\end{tabular}

2) Trabaja en más de un centro de trabajo. En los resultados de la investigación podemos observar que el $70 \%$ de los docentes tiene más de un centro de trabajo, el otro $30 \%$ de los docentes no tiene otro centro de trabajo, es decir solo trabaja en uno. Como se puede apreciar en la tabla 3. 
Tabla 3. Tiene más de un centro de trabajo

\begin{tabular}{lcc}
\hline Centros de trabajo & Frecuencia & $\%$ \\
\hline Tiene dos o más centros de trabajo & 94 & 70 \\
No tiene otro centro de trabajo & 40 & 30 \\
Total & 134 & 100 \\
\hline
\end{tabular}

3) Exigencia ergonómica en dictado de clases, por las múltiples alternativas de las respuestas, el $100 \%$ de los docentes respondieron en este caso las dos, levantar la voz permanentemente en clases, y como también estar sentado y parado, $60 \%$ de los docentes manifestaron que están de pie durante las clases, y un $22 \%$ manifestó que se mueve permanentemente en todo el salón. Como se puede observar en la tabla 4.

Tabla 4. Exigencia ergonómica en dictado de clases

\begin{tabular}{lcc}
\hline Exigencia ergonómica & Frecuencia & $\%$ \\
\hline Estar de pie durante las clases & 80 & 60 \\
Levantar la voz permanentemente en clases & 134 & 100 \\
Moverse permanentemente en todo el salón & 30 & 22 \\
Sentado y parado & 134 & 100 \\
\hline
\end{tabular}

4) Principales enfermedades diagnosticadas, por las múltiples alternativas de respuesta, los resultados del estudio demuestran que las enfermedades diagnosticadas son muy variables, las varices con un $67 \%$ es la enfermedad más común en los docentes, la gastritis con $45 \%$ es la segunda enfermedad más común en los docentes, estas dos enfermedades diagnosticadas son las más relevantes. En la tabla 5 podemos observar todos los resultados.

Tabla 5. Principales enfermedades diagnosticadas

\begin{tabular}{lcc}
\hline Principales enfermedades & Frecuencia & $\%$ \\
\hline Varices & 90 & 67 \\
Disfonía & 45 & 34 \\
Stress & 55 & 41 \\
Gastritis & 60 & 45 \\
Depresión & 38 & 28 \\
Resfriados frecuentes & 14 & 10 \\
Trastornos ginecológicos & 57 & 43 \\
\multicolumn{1}{c}{ Enfermedades de la columna } & 39 & 29 \\
Otros. Epecifique & 25 & 19 \\
\hline
\end{tabular}


5) Resultados de exámenes ocupacionales, los docentes indican que los resultados de sus exámenes ocupacionales son regulares en un $61 \%$ con respecto al año anterior, un $9 \%$ indica que los resultados de sus exámenes ocupacionales son malos con respecto al año anterior, un $26 \%$ indica que los resultados de sus exámenes ocupacionales son buenos con respecto al año anterior, y un solo $4 \%$ indica que los resultados de sus exámenes ocupacionales son óptimos con respecto al año anterior. Como podemos apreciar en la tabla 6.

Tabla 6. Resultados de exámenes ocupacionales

\begin{tabular}{lcc}
\hline Resultado & Frecuencia & $\%$ \\
\hline Optimo & 5 & 4 \\
Bueno & 35 & 26 \\
Regular & 82 & 61 \\
Mal & 12 & 9 \\
Total & 134 & 100 \\
\hline
\end{tabular}

Ahora, haremos un análisis de relaciones entre variables: variables de entrada (causas) y variables de salida (resultados), se está considerado los valores más altos que se han obtenido en cada variable.

Se deduce que la variable alimentación durante el trabajo, en este caso $69 \%$ de docentes su alimentación la realizan en la calle, tiene relación directa con la variable horas trabajadas en exceso durante la semana, el 82 \% manifiesta que trabaja más de 40 horas durante la semana.

También la variable "Razones de trabajar en más de un centro", donde el $90 \%$ de los docentes trabaja en más de un centro de trabajo porque las remuneraciones son bajas, tiene relación con la variable Trabaja en más centro de trabajo donde el $70 \%$ de los docentes indica que tiene más de un centro de trabajo.

La variable "Grado de exigencia del trabajo docente", donde el $54 \%$ considera que el grado de exigencia del trabajo docente es medía, tiene relación con la variable exigencia ergonómica, donde el $100 \%$ de los docentes afirma que tienen que levantar la voz permanentemente durante las clases.

La variable "Edad del docente", donde el $40 \%$ está en el rango de 41 a 50 años, tiene relación con variable resultados de exámenes ocupacionales donde los docentes indican que sus resultados son regulares en un $61 \%$ con respecto al año anterior, y tiene relación a la variable enfermedades diagnósticas donde el $67 \%$ de docentes indican tener varices $(67 \%)$ y un $45 \%$ de docentes indica tener gastritis.

La variable "Descanso entre hora y hora de trabajo", donde el $34 \%$ de docentes indica que descansa menos de 5 minutos entre hora y hora de clases, tiene una relación con la variable principales enfermedades diagnosticadas donde el $67 \%$ de docentes indican tener varices $(67 \%)$ y un $45 \%$ de docentes indica tener gastritis. La "Exigencia ergonómica" durante las clases, donde el $100 \%$ de los docentes afirma que tienen que levantar la voz permanentemente durante las clases. Como se puede apreciar en la tabla 7. 
Tabla 7. Relaciones entre variables (causas y resultados)

\begin{tabular}{|c|c|c|c|c|c|}
\hline Variables (causas) & Frecuencia & $\%$ & Variables (resultados) & Frecuencia & $\%$ \\
\hline Edad del docente & 54 & 40 & $\begin{array}{l}\text { Horas trabajadas durante la } \\
\text { semana }\end{array}$ & 110 & 82 \\
\hline $\begin{array}{l}\text { Descanso entre hora y } \\
\text { hora }\end{array}$ & 45 & 34 & $\begin{array}{l}\text { Trabaja en más centro de } \\
\text { trabajo }\end{array}$ & 94 & 50 \\
\hline $\begin{array}{l}\text { Razones de trabajar en } \\
\text { más de un centro }\end{array}$ & 120 & 90 & $\begin{array}{l}\text { Exigencia ergonómica } \\
\text { durante las clases }\end{array}$ & 134 & 100 \\
\hline Grado de exigencia & 73 & 54 & $\begin{array}{l}\text { Resultados de exámenes } \\
\text { ocupacionales }\end{array}$ & 82 & 61 \\
\hline $\begin{array}{l}\text { Alimentación durante el } \\
\text { trabajo }\end{array}$ & 92 & 69 & $\begin{array}{l}\text { Principales enfermedades } \\
\text { diagnosticadas }\end{array}$ & 90 & 67 \\
\hline
\end{tabular}

\section{DISCUSIÓN}

- Según un estudio de Unesco (3):

Los profesores en un mayor porcentaje (49.6\%) dedican más de 40.1 horas a la realización de tareas docentes, tanto dentro como fuera del trabajo, el $25.5 \%$ de maestros dedican entre 30.1 y 40 horas, y menos de 20 horas el $24.1 \%$ de profesores. Sólo el $0.7 \%$ de profesores trabaja entre 20 y 30 horas.

Nuestro estudio refiere que el $82 \%$ dedica más de 40 horas a la labor docente y el $18 \%$ dedica entre 31 y 40 horas a la labor docente. En relación con el tiempo dedicado al descanso durante la jornada, el $31 \%$ no descansa ni un minuto, el $22 \%$ descansa menos de 5 minutos, el $34 \%$ descansa entre 6 y 15 minutos y el $13 \%$ descansa más de 15 minutos.

- En cuanto a las condiciones de salud, se encuentra que la salud física docente está afectada por una serie de enfermedades diagnosticadas, siendo las varices (67\%), la gastritis (45\%), los trastornos ginecológicos (43\%) y el estrés (41\%) aquellas enfermedades con mayor predominancia. Asimismo, se presentan las siguientes enfermedades en menor medida: disfonía (34\%), enfermedades a la columna (29\%), depresión (28\%), resfriados comunes (10\%).

- También se ha identificado que el $70 \%$ de docentes cuenta con más de un centro de trabajo, y una de las razones porque laboran en dos o más centros de trabajo es por las bajas remuneraciones que perciben, y lo corrobora el resultado de este estudio, donde un categórico $90 \%$ lo confirma. Si bien es cierto que la ley universitaria reciente determina que un docente deba tener como carga horaria 30 horas, la realidad determina que el docente labora en dos o más centro de trabajo donde acumula más horas laboradas y así pueda cubrir su presupuesto familiar.

- Grado de exigencias ergonómica. El estudio detalla que los profesores en un 28\% perciben un "alto" grado de exigencia ergonómica en el trabajo que realizan. Un $54 \%$ percibe en un grado "medio", y un $18 \%$ en un grado "bajo". Las principales causas atribuidas a esta exigencia son el estar de pie durante toda la jornada en un $60 \%$, levantar la voz lo manifiesta un $100 \%$, moverse por todo el salón un 
$22 \%$ y estar parado y sentado durante toda la jornada lo manifiesta el $100 \%$ de los encuestados.

- Según el estudio de Soria y Chiroque (2004) (4) sobre las enfermedades del docente:

El 52.2\% de los docentes refiere haber presentado alguna dolencia o daño a su salud en el año previo, existiendo una mayor frecuencia de enfermedades en los docentes del área rural, lo cual es corroborado por un estudio de la OMS. Las características del trabajo docente predisponen al maestro a presentar ciertas alteraciones en su salud. Algunas acciones físicas del quehacer cotidiano del docente, por ejemplo el esfuerzo vocal y la exposición al polvo de tiza favorecen la aparición de enfermedades que tienen que ver con el aparato fonético.

- Según Fernández (2002) (5) en un investigación realizada en Lima:

El presente estudio explora el síndrome de desgaste psíquico (burnout) en una muestra de 264 profesores de educación primaria de Lima metropolitana. Se analizan las tres dimensiones que comprende el síndrome: agotamiento emocional, despersonalización y falta de realización personal. Los resultados indican que un $43 \%$ de profesores alcanzan niveles altos en el síndrome del desgaste psíquico. Se discuten las diferencias encontradas en cada una de las dimensiones del síndrome en relación con una serie de variables sociodemográficas en las que se encuentra inmerso el maestro.

- Una vez realizado el estudio, podemos concluir que la población docente que labora en dos o más centros de trabajo y con más de 40 horas de carga lectiva, sumados a las condiciones del trabajo, la forma de contratación y demás factores expuestos anteriormente, se encuentran en un nivel alto de exposición a factores de riesgos o daño de su salud, donde se destacan situaciones de vulnerabilidad relacionadas principalmente con una alta carga mental, un mediano riesgo en relación con el contenido específico del trabajo y en lo relacionado con la organización del mismo, pudiendo determinar que las formas de contratación y la realidad que hoy viven las instituciones, afectan las condiciones físicas, biológicas y mentales de los docentes, y que por su injerencia en los resultados de la calidad de los estudiantes que tienen bajo su cargo, ameritan una intervención directa del estado e instituciones educativas privadas que permitan la mitigación de dichos riesgos. La realidad del docente por múltiples necesidades lo obliga a la movilidad entre universidades e institutos, en donde ellos reciban mejores condiciones y garantías laborales; al mismo tiempo las bajas remuneraciones en los centros de estudios les obligan a exponerse. Se recomienda la realización de nuevos estudios en centros de estudios similares para aumentar el conocimiento del agravio de la salud del docente, se requiere crear cultura de prevención en los docentes difundiéndose cartillas de salud docente; la difusión de la presente investigación debe motivar a que los docentes y sus gremios presenten iniciativas legislativas que regulen el exceso de trabajo y se establezcan enfermedades profesionales para el docente.

\section{REFERENCIAS BIBLIOGRÁFICAS}

(1) (3) Oficina Regional de Educación de la UNESCO para América Latina y el Caribe, OREALC/UNESCOSantiagodeChile(2005)Condicionesdetrabajoysaluddocente. 
Recuperado de http://unesdoc.unesco.org/images/0014/001425/142551s.pdf

(2) Enfermedades profesionales, (s.f.). Argentina Sindicato Unificado de Trabajadores de la Educación de Buenos Aires (SUTEBA). Recuperado de http://www.suteba. org.ar/enfermedades-profesionales 669.html

(3) Soria y Chiroque (2004), Salud del Maestro Peruano: Salud Ocupacional Docente. Recuperado de http://repositorio.minedu.gob.pe/bitstream/ handle/123456789/902/532.\%20Salud \%20del \%20maestro \%20peruano \%20 salud \%20ocupacional \%20docente.pdf? sequence $=1$ \&isAllowed $=y$

(4) Fernández, Manuel (2002), Desgaste psíquico (burnout) en profesores de educación primaria de Lima Metropolitana. Recuperado de: http://www.redalyc. org/pdf/1471/147118132002.pdf 